\title{
COVID-19 pathology imaging: A one-year perspective
}

\author{
Martyna Hajac ${ }^{1, A, D, F}$, Cyprian Olchowy ${ }^{2, E, F}$, Rafał Porębaa ${ }^{3, E, F}$, Paweł Gać, ${ }^{1, A, E, F}$ \\ ${ }^{1}$ Department of Hygiene, Wroclaw Medical University, Poland \\ 2 Department of Oral Surgery, Wroclaw Medical University, Poland \\ ${ }^{3}$ Department and Clinic of Internal and Occupational Diseases, Hypertension and Clinical Oncology, Wroclaw Medical University, Poland \\ A - research concept and design; $\mathrm{B}$ - collection and/or assembly of data; $\mathrm{C}$ - data analysis and interpretation; \\ $D$ - writing the article; $E$ - critical revision of the article; $F$ - final approval of the article
}

\section{Address for correspondence \\ Paweł Gać \\ E-mail: pawelgac@interia.pl, pawel.gac@umw.edu.pl}

\section{Funding sources}

None declared

Conflict of interest

None declared

Received on December 29, 2020

Reviewed on January 18, 2021

Accepted on April 15, 2021

Published online on September 30, 2021

Cite as

Hajac M, Olchowy C, Poręba R, Gać P. COVID-19 pathology imaging: A one-year perspective. Dent Med Probl. 2021;58(3):377-384. doi:10.17219/dmp/135814

DOI

$10.17219 / \mathrm{dmp} / 135814$

Copyright

○ 2021 by Wroclaw Medical University

This is an article distributed under the terms of the

Creative Commons Attribution 3.0 Unported License (CC BY 3.0)

(https://creativecommons.org/licenses/by/3.0/).

\begin{abstract}
The first cases of coronavirus disease 2019 (COVID-19) were reported in Wuhan, China, in December 2019. Five months later, the World Health Organization (WHO) announced a pandemic. The symptoms are nonspecific, and include breathing difficulties, cough, fever, and the loss of smell and taste. The diagnosis is confirmed by real-time reverse transcriptase-polymerase chain reaction (RT-PCR) testing. Medical imaging has been mainly used to estimate the range of disease or potential complications.

The aim of this study was to present the radiographic features of COVID-19 reported in published papers. This investigation includes the scientific work concerning chest radiography (chest $X$-ray - CXR) and computed tomography (CT) in COVID-19 patients. The most common pathologies are described, and the classification of COVID-19 appearance in (T and other radiology reports is summarized. The usage of lung ultrasound (LUS) was taken into consideration. This study emphasizes the role of artificial intelligence (Al) in the COVID-19 pandemic. The algorithms developed to detect the disease are discussed. The role of medical imaging is not limited to the respiratory system; it can also be used in searching for and monitoring complications (cardiac, vascular or brain damage). Due to the significant role of radiology in the current pandemic, a review of the latest medical literature was performed to help clarify the upcoming data.
\end{abstract}

Keywords: ultrasound, computed tomography, radiography, COVID-19, artificial intelligence 


\section{Introduction}

Coronavirus disease 2019 (COVID-19) is a medical condition caused by severe acute respiratory syndrome coronavirus 2 (SARS-CoV-2). In March 2020, the World Health Organization (WHO) declared the disease a pandemic. ${ }^{1}$ Globally, as of December 2020, the number of COVID-19 cases was over 75 million and so far the disease has affected almost every territory. Worldwide, the current number of deaths exceeds 1.6 million. ${ }^{2}$ The symptoms and signs of the infection are non-specific, and may include fever, cough, anosmia, and fatigue. Coronavirus disease 2019 mainly affects the respiratory system; therefore, the role of thoracic radiology should be established. The COVID-19 diagnosis is based on the real-time reverse transcriptasepolymerase chain reaction (RT-PCR) test. ${ }^{3}$ Due to the symptoms of pneumonia, some patients require medical imaging. This study aimed to systematize the radiographic features presented in a traditional radiograph, computed tomography (CT) and lung ultrasound (LUS). Based on medical imaging data, many artificial intelligence (AI) algorithms have been developed to help in the detection of COVID-19. During the last year, multiple articles highlighting the radiological findings in COVID-19 patients were published. Many of these studies focused on a particular method of imaging or a specific type of disease complication. The role of this study was to systematize these results and conclusions for optimal use in an everyday clinical setting.

\section{Methodology}

The goal of this article was to provide an overview of COVID-19 pathology imaging. A review of all the relevant literature published in 2020 as well as of the current statements of the European Society of Radiology (ESR), the Radiological Society of North America (RSNA) and the British Society of Thoracic Imaging (BSTI) were performed. The sources of the utilized data were PubMed and Google Scholar databases. Only articles in English were included.

\section{Chest radiography}

Chest radiography (chest X-ray - CXR) is less sensitive than chest CT. However, due to the limited availability and high costs of CT, CXR is performed first. Furthermore, in some countries, CXR cannot be followed up by $\mathrm{CT}$, especially during a pandemic. For this reason, it is essential to describe the radiographic features which can be seen on a chest radiograph, not only by a radiologist, but also other specialists. ${ }^{4}$ Chest radiography in early and mild cases of COVID-19 may

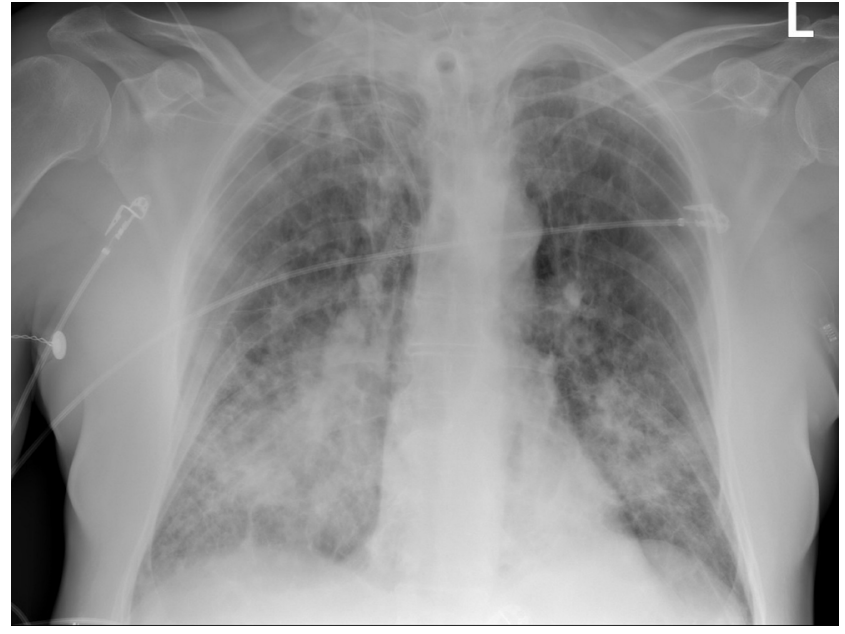

Fig. 1. Chest radiography (CXR). Bilateral pneumonia in a patient with confirmed coronavirus disease 2019 (COVID-19)

not reveal any pathologies. Studies from China showed that only $54.2 \%$ of non-severe disease cases presented with abnormalities on chest radiographs; in severe cases, almost $77 \%$ of patients had an abnormal chest

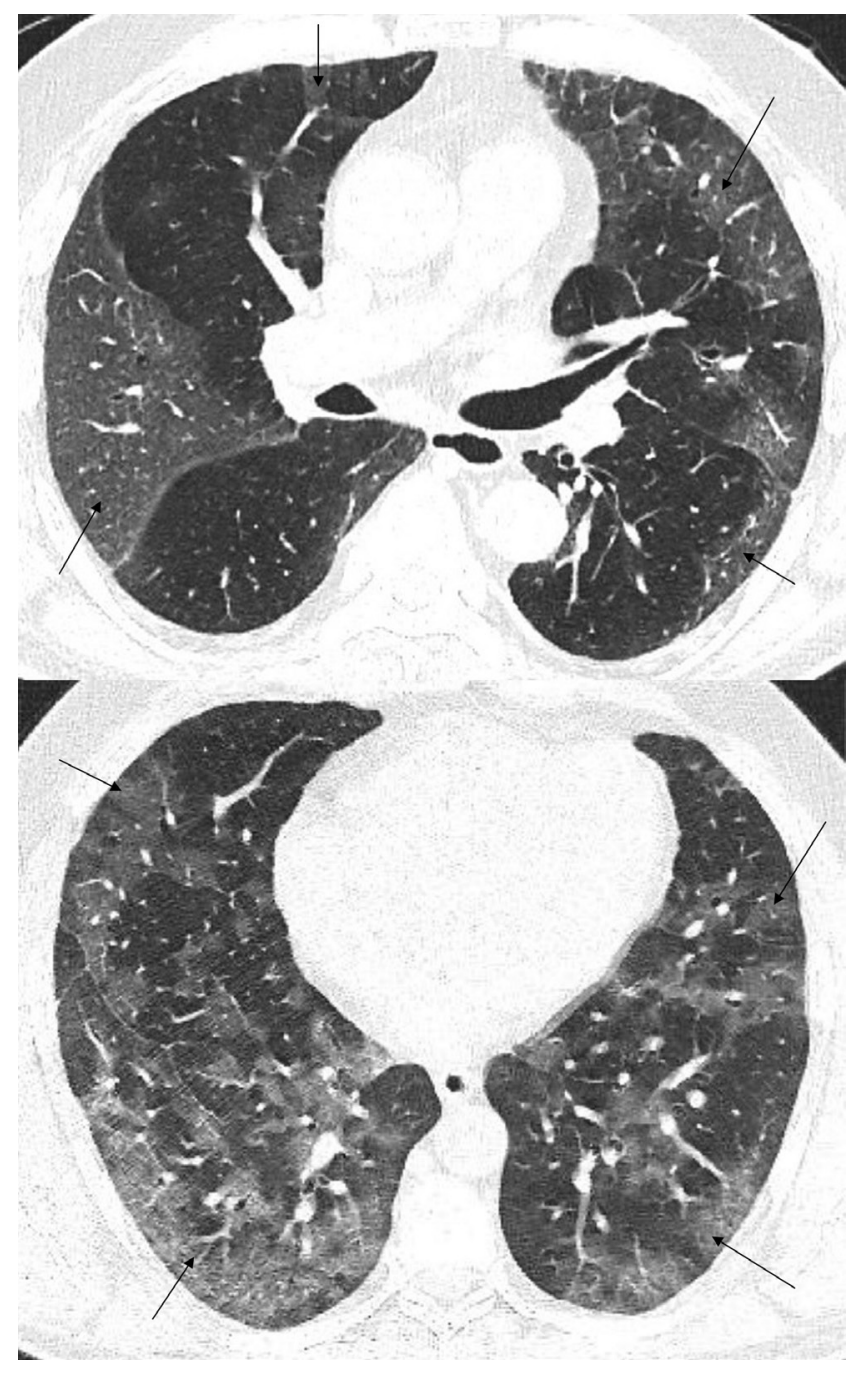

Fig. 2. Chest computed tomography (CT). Ground-glass opacities (GGOs) (arrows) in a patient with confirmed COVID-19 
radiograph. ${ }^{5}$ The most common radiographic features are patchy or diffuse asymmetric air-space opacities, consolidations, and ground-glass opacities (GGOs). ${ }^{6}$ The distribution of these features is frequently bilateral. The peripheral and lower zone distribution are the prevalent locations of these signs (Fig. 1). Pleural effusion is a rare complication experienced by approx. $3 \%$ of patients. In these cases, there is no pathognomonic appearance of COVID-19 in the chest radiograph and the image is similar to other pneumonias. ${ }^{4}$ Due to this fact, CXR is not recommended for screening or use as a diagnostic tool for COVID-19. It should be noted that portable imaging equipment limits the transportation of patients and minimizes the risk of spreading the disease. ${ }^{7}$ Reports from radiology departments, including the University of Washington and Australian hospitals, highlighted a technique whereby an X-ray can be performed through a glass window and the patient does not leave the isolation room. ${ }^{8,9}$

\section{Chest computed tomography}

In the early stage of COVID-19, the primary CT findings observed are bilateral GGOs with a mostly peripheral and subpleural location. Ground-glass opacity is a term used to describe hazy areas with the

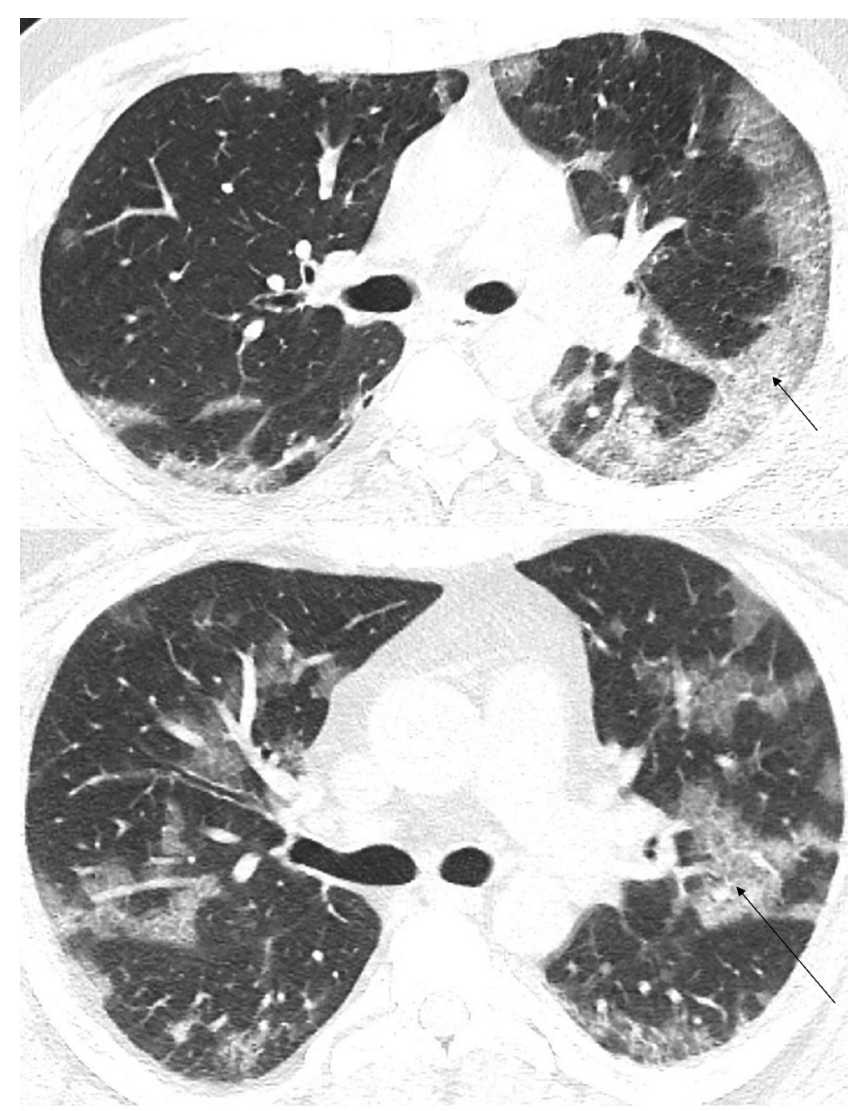

Fig. 3. Chest CT. Crazy-paving appearance (arrows) in a patient with confirmed COVID-19

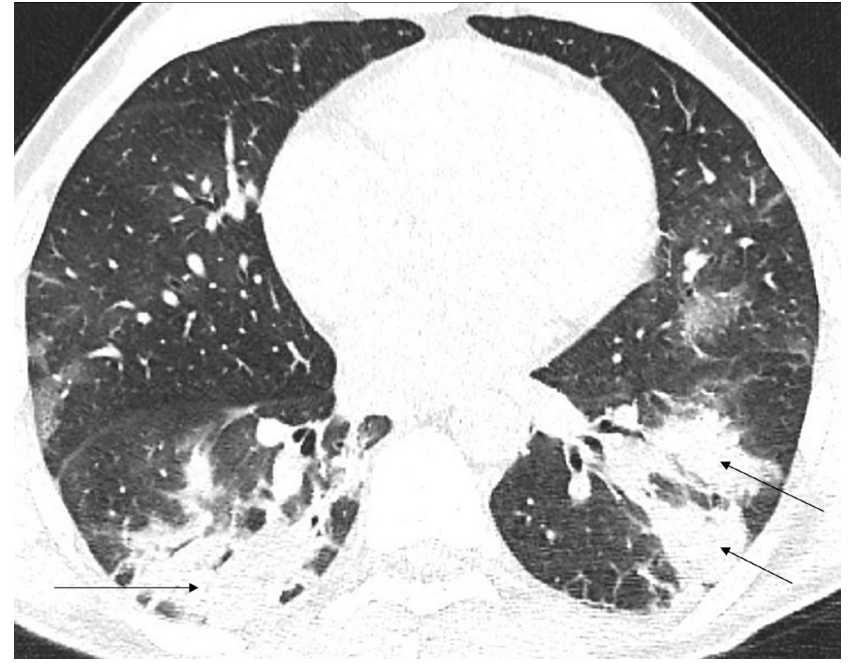

Fig. 4. Chest CT. Consolidations (arrows) in a patient with confirmed COVID-19

preservation of vascular and bronchial margins which are a result of the partial filling of air spaces, intestinal thickening, or the coexistence of both (Fig. 2). ${ }^{10}$ Another phenomenon is described as having 'crazypaving' appearance. This is a combination of GGO and interlobular or intralobular septal thickening (Fig. 3). This condition is mostly present in severe cases of COVID-19 pneumonia. Consolidations (multifocal, patchy or segmental) are another sign of COVID-19 progression (Fig. 4). ${ }^{11}$ Several days after developing the first symptoms, chest CT can reveal linear consolidations and reversed halo signs in the patient's lungs. ${ }^{12} \mathrm{Ra}-$ diographic features typically include bronchovascular thickening (Fig. 5). ${ }^{13}$

Recently, COVID-19 progression has been described with successive CT scans and has been correlated with the onset of symptoms. Shi et al. monitored a patient for 3 weeks and performed $4 \mathrm{CT}$ scans. ${ }^{14}$ The $1^{\text {st }} \mathrm{CT}$ scan,

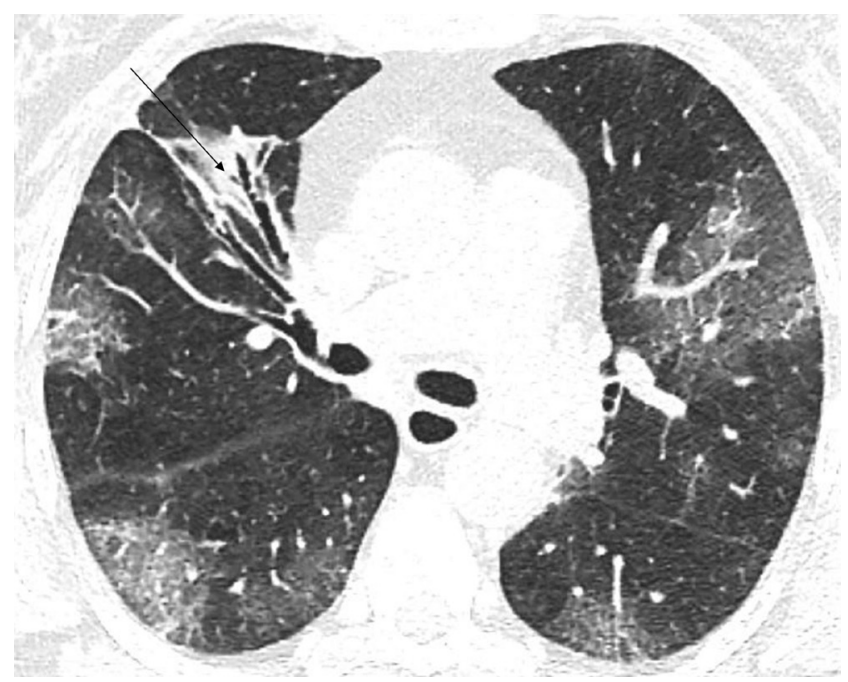

Fig. 5. Chest CT. Bronchovascular thickening (arrow) in a patient with confirmed COVID-19 
obtained 9 days after the onset of symptoms, revealed multifocal bilateral GGOs. After 15 days of disease evolution, a mixed pattern of GGOs and consolidations was observed. The signs of healing were seen after 19 days. The last CT scan was performed on day 31 and no abnormalities were found. ${ }^{14}$

A retrospective study of 121 symptomatic patients infected with SARS-CoV-2 was performed by Bernheim et al. ${ }^{15}$ The main aim of their research was to assess the correlation between the onset of symptoms and common findings on the initial CT scan. The time between the occurrence of the first symptoms and subsequent CT scans was divided into 3 periods: early (0-2 days); intermediate ( $3-5$ days); and late (6-12 days). Bilateral lung evolvement, which is considered typical in COVID-19 pneumonia, was observed in $28 \%$ of earlystage patients, $76 \%$ of intermediate-stage ones and $88 \%$ of late-stage patients. Crazy-paving appearance and the reversed halo effect were absent in the early group, but were mostly present in the late-stage group. It was found that $56 \%$ of the early patients showed the lack of lung capacities during the radiological examination despite the presence of clinical symptoms. The study results exhibit imaging patterns, essential not only for understanding the pathophysiology or natural history of COVID-19, but also for the prediction of its progression and potential complications. ${ }^{15}$

Based on similar research, 4 stages of CT imaging have been established. The $1^{\text {st }}$ stage is named the early or initial stage and lasts up to 4 days. A CT scan performed during this period may not reveal any pathologies or may only indicate the presence of GGOs. It is crucial to remember that up to half of patients may have a normal CT scan 2 days after the symptoms occur. The next phase is the progressive stage (5-8 days) - several GGOs arise and the crazy-paving pattern becomes vis-

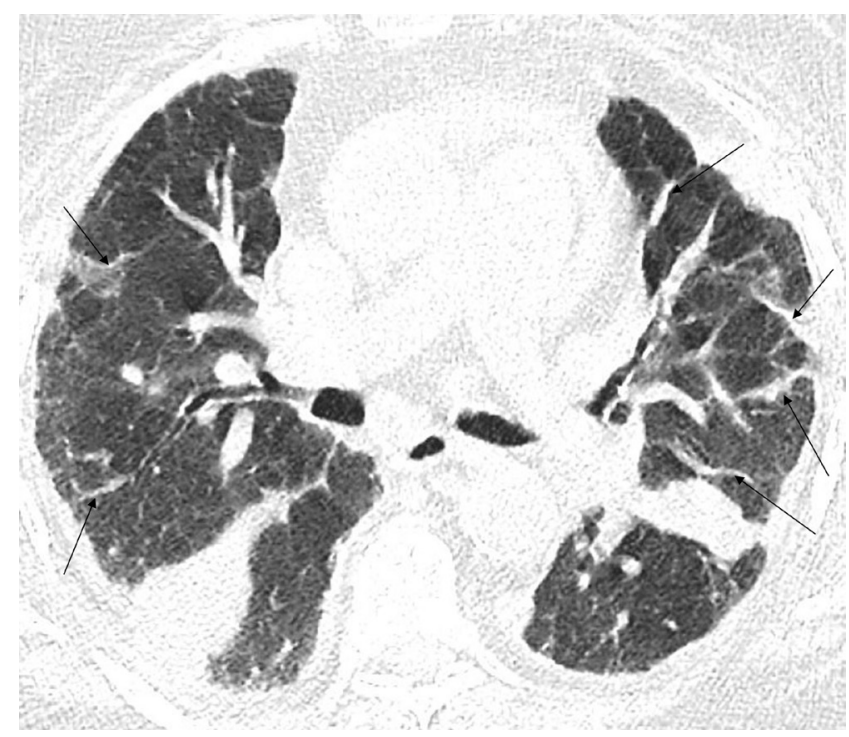

Fig. 6. Chest CT. Fibrous stripes (arrows) in a patient with confirmed COVID-19 ible. Between 9 and 13 days, consolidations appear. This phase is called the peak stage. After 14 days, absorption begins and fibrous stripes may occur (Fig. 6). After at least 1 month, and if no other complications arise, the patient's lungs are able to return to a condition similar to that before COVID-19. ${ }^{16}$

\section{Tomographic reporting methods and CO-RADS}

Due to the never-before-seen scale of pneumonia and the rising amount of $\mathrm{CT}$ scans, creating a standardized reporting language for radiologists has become necessary. The BSTI published a report with suggestions regarding plain radiography. ${ }^{17}$ In cooperation, the RSNA, the American College of Radiology (ACR), and the Society of Thoracic Radiology (STR) released a special statement. ${ }^{17}$ The statement aimed to present a classification of COVID-19 appearance derived from CT. This classification suggests 4 types of images: typical; indeterminate; atypical; or negative for pneumonia. The typical appearance includes GGOs, consolidations, the crazy-paving pattern, and the reverse halo effect. Pathologies are located peripherally and bilaterally. The indeterminate appearance is characterized by the absence of typical CT findings, but the presence of other pathologies, including multifocal, diffuse, peripheral, or unilateral GGOs with or without consolidations, and a few small GGOs with non-rounded and non-peripheral distribution. The atypical appearance does not consist of typical or indeterminate features, but includes yet other pathologies. In this category, the presence of isolated lobar or segmental consolidations, without GGOs, is acceptable. Lung cavitation, discrete small nodules and smoother interlobular septal thickening with pleural effusion may occur. Regarding the last category, these include patients with no signs of COVID-19 pneumonia. ${ }^{17}$ This chest CT classification is widely used for clinical practice and subsequent studies. ${ }^{18}$ The Dutch Association for Radiology introduced a dedicated COVID-19 scoring system. ${ }^{18}$ It was named CO-RADS as a short version for COVID-19 Reporting and Data System. The role of this classification is to estimate the suspicion of COVID-19 pneumonia. Number 1 is reserved for a lack of probability. Higher numbers (up to 5) refer to typical COVID-19 features. CO-RADS 0 describes an incomplete examination or insufficient quality of CT. CO-RADS 6 is associated with a confirmed positive RT-PCR test.

Nowadays, various radiological societies and organizations remind us that CT should not be included in diagnostic and screening tools for COVID-19. Computed tomography scans should be reserved for patients in a serious condition to better estimate the stage of the disease as well as its progression or potential complications, and if RT-PCR is currently unavailable. ${ }^{19}$ 


\section{Chest ultrasound}

Lung ultrasound has recently become the recommended examination for acute respiratory failure. ${ }^{20}$ In COVID-19 patients, early investigations from China suggest the promising utility of LUS in a 12-zone method. ${ }^{21}$ Characteristic findings included various forms of B-lines (focal, multifocal and confluent). The LUS of many patients with COVID-19 pneumonia revealed an unusual artifact. It has been described as a shining band-form artifact arising from a regular pleural line. ${ }^{21}$ The first Chinese researchers compared this artifact to a waterfall. In further scientific works, the term "waterfall" was replaced with "light-beam sign".22 This artifact correlates with GGOs, which can be seen on CT scans. ${ }^{23}$ Lung ultrasound can also a reveal a thickened and irregular pleural line. Ultrasonographic features include various types of consolidations - multifocal, both non-translobar and translobar with air bronchograms. Pleural effusion may be observed during the examination, but it is not a common pathology. The observed features are related to the stage of the disease and change along with its duration. In early or mild infections, focal B-lines are frequently present. The LUS of COVID-19 patients in a critical condition shows alveolar intestinal syndrome (AIS). A-lines appear during recovery and uneven B-lines can occur as a sign of pulmonary fibrosis. Lung ultrasound can have a crucial role in the diagnosis and monitoring of patients with COVID-19. The main advantages are safety and the lack of radiation. This means unlimited access, due to the lack of exposure to ionizing radiation on one hand, and economic reasons on the other hand, and repeatability. Moreover, examinations can also be performed daily. ${ }^{24}$ In addition, the patient avoids potentially dangerous transport to the radiology department.

\section{Complications}

COVID-19 may result in a number of complications ranging from mild to very severe cases. It is estimated that $17-29 \%$ of patients develop acute respiratory distress syndrome (ARDS). ${ }^{6}$ Although the disease mostly affects the respiratory system, complications may influence any other system. Commonly reported sequelae are acute cardiac injuries. The mechanism of cardiovascular injury from COVID-19 has not been completely explained and could be a complex phenomenon. However, it manifests itself by myocardial ischemia and cardiac arrest. Recent works describe not only elevated troponin levels, but also myocardial ischemia and cardiac arrest associated with COVID-19 infection. ${ }^{25}$ The authors of the editorial entitled "Coronavirus disease 2019 (COVID-19) and the heart - Is heart failure the next chapter?" point to various cardiac complications after pneumonia. ${ }^{26}$ The diagnosis of cardiovascular disease (CVD) complications should be based on examination, symptoms, elevated cardiac markers, and electrocardiography (ECG). However, when the patient's condition is not stable, further steps must be taken, including medical imaging ${ }^{27}$ (e.g., a CT $\operatorname{scan}^{28}$ ). A severe COVID-19 complication is myocarditis (Fig. 7). Puntmann et al. suggest that approx. $60 \%$ of patients could experience myocardial inflammation, with no association to preexisting conditions or the severity of COVID-19. ${ }^{29}$ When myocarditis is suspected, the examination that should be taken into consideration is cardiac magnetic resonance (CMR). ${ }^{29}$ It is confirmed that SARS-CoV-2 infection increases the risk of pulmonary embolism (Fig. 8) and deep vein thrombosis. ${ }^{30}$ When acute thromboembolic disease is suspected, not only should D-dimer levels be tested, but an assessment via computed tomography angiography (CTA) is essential. ${ }^{31}$

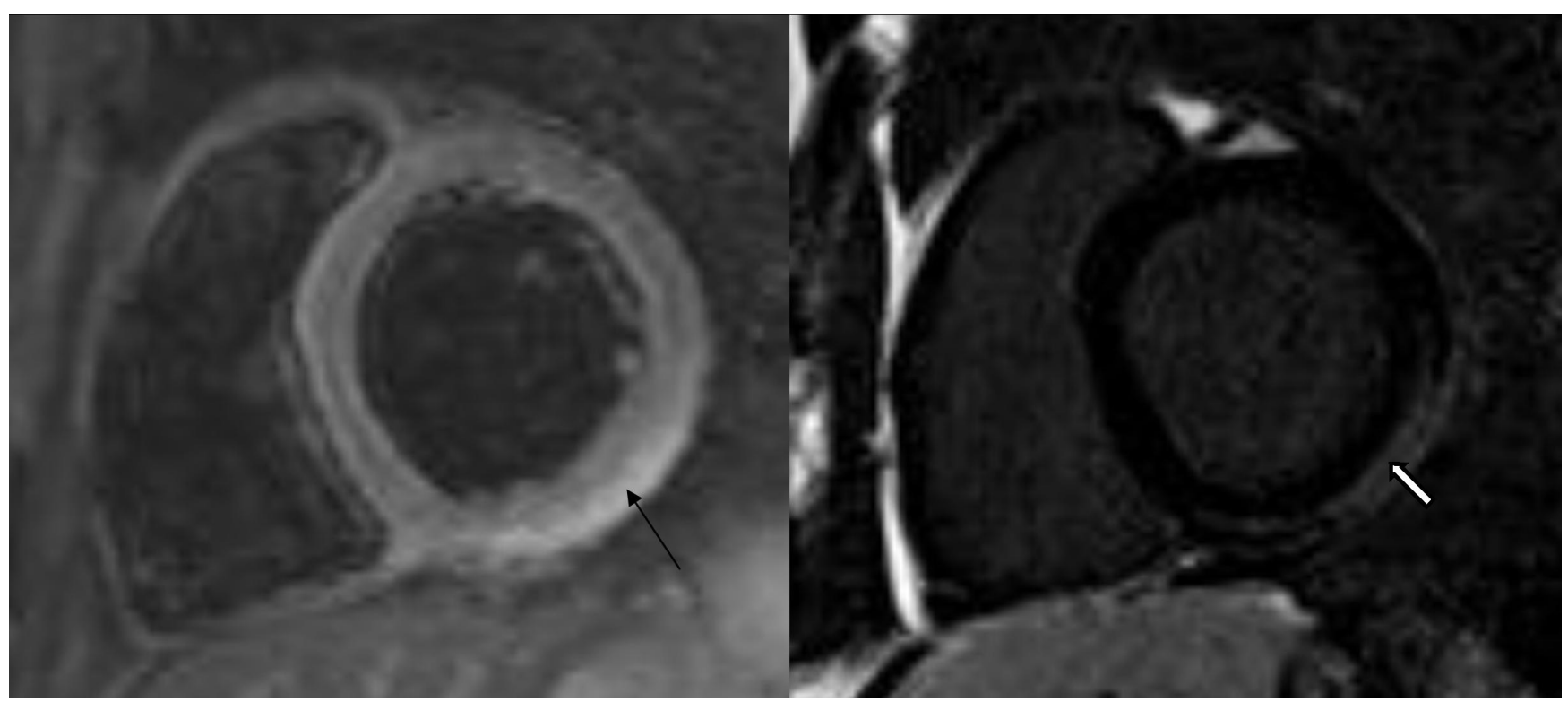

Fig. 7. Cardiac magnetic resonance (CMR). Myocardial edema in the STIR sequence (black arrow) and the area of late gadolinium enhancement in the LGE sequence (contour of black arrow) during active myocarditis 


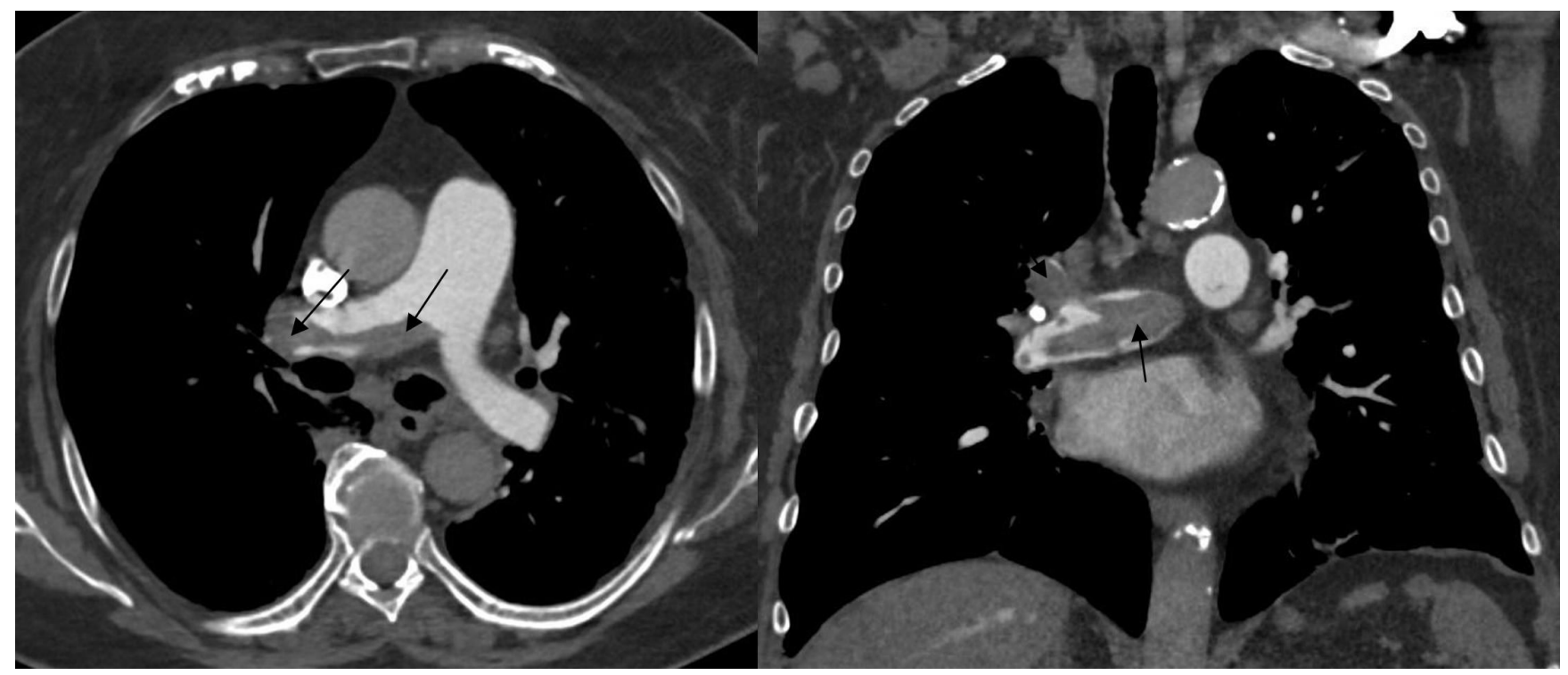

Fig. 8. Pulmonary computed tomography angiography (CTA). Loss of pulmonary artery filling (arrows) during acute pulmonary embolism

COVID-19 patients are at an increased risk of brain tissue damage. The injury of heart or brain tissue can be caused via 4 different pathways. These include the neuronal pathway, the hypoxia pathway, the renin-angiotensin-aldosterone system (RAAS) pathway, and the immune pathway. Patients with comorbidities are a highrisk group and need to be kept under careful observation. In case brain hemorrhage is suspected, CT or magnetic resonance imaging (MRI) should be performed (Fig. 9). ${ }^{32}$ Medical imaging is an essential tool in screening and localizing life-threatening COVID-19.

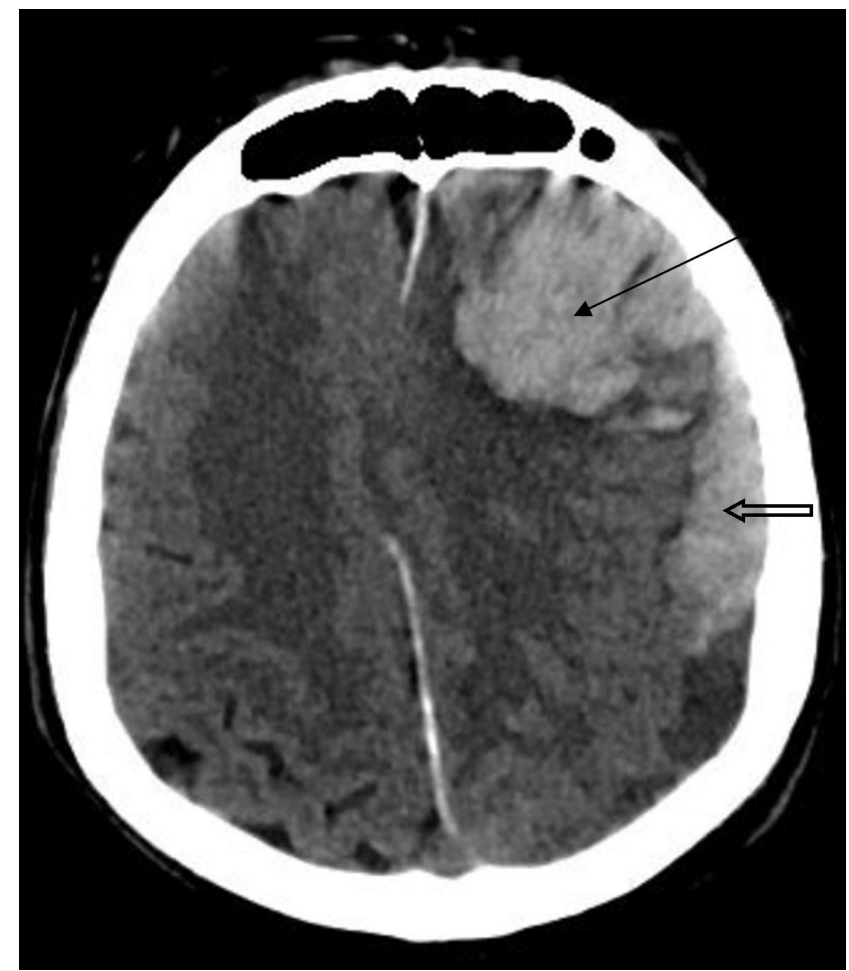

Fig. 9. Head CT. Intracranial hyperdense foci corresponding to hemorrhagic stroke (black arrow) and cerebral hematoma (contour of black arrow)

\section{Artificial intelligence}

The global pandemic has forced the medical industry to search for new technologies to estimate and control the spread of the new virus. During periods of peak infection, global healthcare infrastructure deteriorated rapidly. The main issue was staff shortage in many departments, including radiology. When the scale of the problem became unmeasurable, medical organizations decided to use a potentially vital, but still unexplored area of science, i.e., artificial intelligence (AI). ${ }^{33}$ The global crisis allowed AI to be implemented in many facets of healthcare and epidemiology at the same time. These applications included tracking the spread of the virus or identifying high-risk patients. ${ }^{34}$ However, due to the lack of radiologists, few programs using AI to diagnose COVID-19 were developed.

The main tasks for AI applications in radiology are the accurate detection of COVID-19 based on CXR or CT images, the differentiation of COVID-19 pneumonia from other kinds of pneumonia, and the assessment of the severity of the process in correlation with the patient's clinical status. Programs based on several pre-trained networks gained high diagnostic sensitivity and specificity, ranging from $84 \%$ to $100 \%$ and from $71 \%$ to $100 \%$, respectively. ${ }^{35}$ The high specificity for the differentiation of diagnosis between COVID-19 and non-COVID19 pneumonia via $\mathrm{AI}$ is promising. ${ }^{36}$ In most cases, the algorithms were based on deep neural network learning, whilst some were based on machine learning with the use of thousands of radiologic images as a comparator. However, these data are heterogeneous due to the fact that they were obtained from different medical units, with the application of different equipment and slightly different protocols. Despite the encouraging results, the AI-based diagnosis of radiographic evidence should be considered as early data that 
requires validation and further standardization before it can be used in clinical practice. ${ }^{35}$

Although the role of AI in medicine is constantly developing, some of the benefits can already be highlighted. Doctors are limited with time; on the other hand, AI technologies can process an incomparable amount of data (i.a., X-ray scans) in a short period. In addition, the global machine learning research community enables sharing the data and innovations. As the pandemic develops and the disease spreads all over the world, access to up-todate information is vital. ${ }^{37}$

Artificial intelligence allows the processing of various types of information from the patient's medical history. It can correlate data like medical imaging, laboratory results, and clinical observations to conclude the most probable course of the disease and suggest further actions. When access to medical services is limited, systems based on telehealth may help. Patients can receive the help they require without leaving their homes, which stops the spread of the virus. Artificial intelligence may provide preliminary healthcare education and advice to COVID-19 patients. Additionally, it can inform on preventive measures. ${ }^{38}$

\section{Summary}

Typical medical imaging in COVID-19 patients includes CXR, CT and LUS. Chest radiography should not be used for the diagnosis and screening of COVID-19. However, it may be performed in severe cases of the disease. The most common findings are patchy or diffuse asymmetric air spaces, consolidations, and GGOs. Similar pathologies can be described in chest CT. Previously, $\mathrm{CT}$ was used to monitor the condition of patients. In the first stage of the disease, any pathologies may not be revealed; however, after a few days (up to 8 days), GGOs and the crazy-paving pattern are the most common signs. Next, consolidations become visible. During the last period of COVID-19, fibrous stripes appear. Computed tomography scans are useful to estimate the range of disease, its progression and any complications. Lung ultrasound is a tool that is recommended for monitoring patients and has a growing role in diagnosis. Focal B-lines are present in early infection and A-lines are visualized during recovery; a thickened and irregular pleural line, various types of consolidations and an artifact called a light-beam sign may be also seen. The role of radiology is not only limited to the imaging of the lungs, but it can also be used in other organs. Computed tomography angiography is performed when acute thromboembolic disease occurs. Cardiovascular magnetic resonance is another useful tool to assess cardiac complications. When brain tissue damage is suspected, MRI is essential. Medical imaging is irreplaceable in the diagnosis of COVID-19-positive patients; however, it is essential to perform it in a specific and directed situation. This study aimed to set in order the most common COVID-19 imaging methods, but also to match the appropriate imaging method to specific patient cases. It is crucial to perform an appropriate test when it is needed (e.g., in case of possible COVID-19 complications). It is also necessary to note that X-ray exposure should be avoided, as in $\mathrm{CO}$ VID-19 screening, CXR is not recommended, but is still performed in many medical wards. Lastly, AI is emerging as a new healthcare solution that is capable of analyzing large amounts of data. Furthermore, AI can correlate this data with the imaging data from the clinic.

\section{ORCID iDs}

Martyna Hajac (D) https://orcid.org/0000-0001-9271-7401

Cyprian Olchowy (D) https://orcid.org/0000-0002-6559-3973

Rafał Poręba (i) https://orcid.org/0000-0002-7217-7070

Paweł Gać (D) https://orcid.org/0000-0001-8366-0239

\section{References}

1. Harapan H, Itoh N, Yufika A, et al. Coronavirus disease 2019 (COVID-19): A literature review. J Infect Public Health. 2020;13(5):667-673. doi:10.1016/j.jiph.2020.03.019

2. World Health Organization. COVID-19 Weekly Epidemiological Update. October 12, 2020. https://www.who.int/docs/default-source/ coronaviruse/situation-reports/20201012-weekly-epi-update-9.pdf.

3. Naserghandi A, Allameh SF, Saffarpour R. All about COVID-19 in brief. New Microbes New Infect. 2020;35:100678. doi:10.1016/j. nmni.2020.100678

4. Wong HYF, Lam HYS, Fong AHT, et al. Frequency and distribution of chest radiographic findings in patients positive for COVID-19. Radiology. 2020;296(2):E72-E78. doi:10.1148/radiol.2020201160

5. Guan W, Ni Z, Hu Y, et al. Clinical characteristics of coronavirus disease 2019 in China. N Eng J Med. 2020;382:1708-1720. doi:10.1056/NEJMoa2002032

6. Rodrigues JCL, Hare SS, Edey A, et al. An update on COVID-19 for the radiologist - a British Society of Thoracic Imaging statement. Clin Radiol. 2020;75(5):323-325. doi:10.1016/j.crad.2020.03.003

7. Kooraki S, Hosseiny M, Myers L, Gholamrezanezhad A. Coronavirus (COVID-19) outbreak: What the Department of Radiology should know. J Am Coll Radiol. 2020;17(4):447-451. doi:10.1016/j.jacr.2020.02.008

8. Mossa-Basha M, Medverd J, Linnau KF, et al. Policies and guidelines for COVID-19 preparedness: Experiences from the University of Washington. Radiology. 2020;296(2):E26-E31. doi:10.1148/radiol.2019201326

9. Brady Z, Scoullar H, Grinsted B, et al. Technique, radiation safety and image quality for chest X-ray imaging through glass and in mobile settings during the COVID-19 pandemic. Phys Eng Sci Med. 2020;43(3):765-779. doi:10.1007/s13246-020-00899-8

10. Hansell DM, Bankier AA, MacMahon H, McLoud TC, Müller NL, Remy J. Fleischner Society: Glossary of terms for thoracic imaging. Radiology. 2008;246(3):697-722. doi:10.1148/radiol.2462070712

11. Ye Z, Zhang Y, Wang Y, Huang Z, Song B. Chest CT manifestations of new coronavirus disease 2019 (COVID-19): A pictorial review. Eur Radiol. 2020;30(8):4381-4389. doi:10.1007/s00330-020-06801-0

12. Revel MP, Parkar AP, Prosch H, et al.; European Society of Radiology (ESR) and the European Society of Thoracic Imaging (ESTI). COVID-19 patients and the radiology department - advice from the European Society of Radiology (ESR) and the European Society of Thoracic Imaging (ESTI). Eur Radiol. 2020;30(9):4903-4909. doi:10.1007/s00330-020-06865-y

13. Kaufman AE, Naidu S, Ramachandran S, Kaufman DS, Fayad ZA, Mani V. Review of radiographic findings in COVID-19. World J Radiol. 2020;12(8):142-155. doi:10.4329/wjr.v12.i8.142

14. Shi $H, H a n X$, Zheng C. Evolution of CT manifestations in a patient recovered from 2019 novel coronavirus (2019-nCoV) pneumonia in Wuhan, China. Radiology. 2020;295(1):20. doi:10.1148/radiol.2020200269 
15. Bernheim A, Mei X, Huang $M$, et al. Chest $C T$ findings in coronavirus disease-19 (COVID-19): Relationship to duration of infection. Radiology. 2020;295(3):200463. doi:10.1148/radiol.2020200463.

16. Pan F, Ye T, Sun $P$, et al. Time course of lung changes on chest CT during recovery from coronavirus disease 2019 (COVID-19). Radiology. 2020;295(3):715-721. doi:10.1148/radiol.2020200370

17. Simpson S, Kay FU, Abbara S, et al. Radiological Society of North America expert consensus statement on reporting chest $\mathrm{CT}$ findings related to COVID-19. Endorsed by the Society of Thoracic Radiology, the American College of Radiology, and RSNA - Secondary Publication. J Thorac Imaging. 2020;35(4):219-227. doi:10.1097/RTI.0000000000000524

18. de Jaegere TMH, Krdzalic J, Fasen BACM, Kwe RM. Radiological Society of North America chest CT classification system for reporting COVID-19 pneumonia: Interobserver variability and correlation with reverse-transcription polymerase chain reaction. Radiol Cardiothorac Imaging. 2020;2(3):e200213. doi:10.1148/ryct.2020200213

19. British Society of Thoracic Imaging. Thoracic imaging in COVID-19 infection. Guidance for the Reporting Radiologist. Version 2. March 16, 2020. https://www.bsti.org.uk/media/resources/files/BSTI_COVID-19_ Radiology_Guidance_version_2_16.03.20.pdf.

20. Mongodi S, Pozzi M, Orlando A, et al. Lung ultrasound for daily monitoring of ARDS patients on extracorporeal membrane oxygenation: Preliminary experience. Intensive Care Med. 2018;44(1):123-124. doi:10.1007/s00134-017-4941-7

21. Volpicelli G, Gargani L. Sonographic signs and patterns of COVID-19 pneumonia. Ultrasound J.2020;12(1):20. doi:10.1186/s13089-020-00171-W

22. Huang $Y$, Wang $S$, Liu $Y$, et al. A preliminary study on the ultrasonic manifestations of peripulmonary lesions of non-critical novel coronavirus pneumonia (COVID-19). Research Square; preprint. 2020. doi:10.21203/rs.2.24369/v1

23. Volpicelli G, Lamorte A, Villén T. What's new in lung ultrasound during the COVID-19 pandemic. Intensive Care Med. 2020;46(7):1445-1448. doi:10.1007/s00134-020-06048-9

24. Peng QY, Wang XT, Zhang LN; Chinese Critical Care Ultrasound Study Group (CCUSG). Findings of lung ultrasonography of novel corona virus pneumonia during the 2019-2020 epidemic. Intensive Care Med. 2020;46(5):849-850. doi:10.1007/s00134-020-05996-6

25. Long B, Brady WJ, Koyfman A, Gottlieb M. Cardiovascular complications in COVID-19. Am J Emerg Med. 2020;38(7):1504-1507. doi:10.1016/j.ajem.2020.04.048

26. Yancy CW, Fonarow GC. Coronavirus disease 2019 (COVID-19) and the heart - Is heart failure the next chapter? JAMA Cardiol. 2020;5(11):1216-1217. doi:10.1001/jamacardio.2020.3575

27. Kang $Y$, Chen T, Mui D, et al. Cardiovascular manifestations and treatment considerations in COVID-19. Heart. 2020;106(15):1132-1141. doi:10.1136/heartjnl-2020-317056

28. Hu H, Ma F, Wei X, Fang Y. Coronavirus fulminant myocarditis treated with glucocorticoid and human immunoglobulin. Eur Heart J. 2021;42(2):206. doi:10.1093/eurheartj/ehaa190

29. Puntmann VO, Carerj ML, Wieters I, et al. Outcomes of cardiovascular magnetic resonance imaging in patients recently recovered from coronavirus disease 2019 (COVID-19). JAMA Cardiol. 2020;5(11):1265-1273. doi:10.1001/jamacardio.2020.3557

30. Klok FA, Kruip MJHA, van der Meer NJM, et al. Confirmation of the high cumulative incidence of thrombotic complications in critically ill ICU patients with COVID-19: An updated analysis. Thromb Res. 2020;191:148-150. doi:10.1016/j.thromres.2020.04.041

31. Léonard-Lorant I, Delabranche X, Séverac F, et al. Acute pulmonary embolism in patients with COVID-19 at CT angiography and relationship to d-dimer levels. Radiology. 2020;296(3):E189-E191. doi:10.1148/radiol.2020201561

32. Suri JS, Puvvula A, Biswas M, et al. COVID-19 pathways for brain and heart injury in comorbidity patients: A role of medical imaging and artificial intelligence-based COVID severity classification: A review. Comput Biol Med. 2020;124:103960. doi:10.1016/j.compbiomed.2020.103960

33. Laudanski K, Shea G, DiMeglio M, Restrepo M, Solomon C. What can COVID-19 teach us about using $\mathrm{Al}$ in pandemics? Healthcare (Basel). 2020;8(4):527. doi:10.3390/healthcare8040527

34. Vaishya $R$, Javaid $M$, Khan IH, Haleem A. Artificial intelligence (AI) applications for COVID-19 pandemic. Diabetes Metab Syndr. 2020;14(4):337-339. doi:10.1016/j.dsx.2020.04.012
35. Ozsahin I, Sekeroglu B, Musa MS, Mustapha MT, Ozsahin DU. Review on diagnosis of COVID-19 from chest CT images using artificial intelligence. Comput Math Methods Med. 2020;2020:9756518. doi:10.1155/2020/9756518

36. Khan Al, Shah JL, Bhat MM. CoroNet: A deep neural network for detection and diagnosis of COVID-19 from chest x-ray images. Comput Methods Programs Biomed. 2020;196:105581. doi:10.1016/j.cmpb.2020.105581

37. Desai SB, Pareek A, Lungren MP. Deep learning and its role in COVID-19 medical imaging. Intell Based Med. 2020;3:100013. doi:10.1016/j.ibmed.2020.100013

38. Tayarani NMH. Applications of artificial intelligence in battling against COVID-19: A literature review. Chaos Solitons Fractals. 2021;142:110338. doi:10.1016/j.chaos.2020.110338 